\title{
Chaotic Properties of the Arctic Oscillation Index
}

\author{
Yoshito Hirata $^{1}$, Yuko Shimo ${ }^{2}$, Hiroshi L. Tanaka ${ }^{3}$, and Kazuyuki Aihara ${ }^{1}$ \\ ${ }^{1}$ Institute of Industrial Science, The University of Tokyo, Tokyo, Japan \\ ${ }^{2}$ Graduate School of Life and Environmental Sciences, University of Tsukuba, Tsukuba, Japan \\ ${ }^{3}$ Center for Computational Sciences, University of Tsukuba, Tsukuba, Japan
}

\begin{abstract}
In this letter, we investigate nonlinear properties of the underlying dynamics for the Arctic Oscillation Index (AOI). First, we develop a test statistic for surrogate data analysis so that we can deal with the high-dimensionality of AOI. Second, we validate the test statistic with toy models. Then, by applying the proposed new test statistic with surrogate data to the dataset of AOI for the last 60 years, we show that AOI is nonlinear with determinism beyond pseudo-periodicity. These results mean that the underlying dynamics of AOI is consistent with deterministic chaos, implying that it is predictable in a short-term but not in a long-term.
\end{abstract}

\section{Introduction}

The recent global warming is a serious problem all over the world. To understand the mechanism of the global warming better, we need to understand weekly and/or seasonal variability of the weather better.

It was 27 years ago that Nicolis and Nicolis (1984) suggested the existence of a climate attractor by estimating the correlation dimension based on the oxygen isotope record obtained from the deep-sea core. Since then, it has been debated whether climate and weather attractors exist or not by using various types of datasets (Grassberger 1986; Essex et al. 1987; Nicolis and Nicolis 1987; Grassberger 1987; Tsonis and Elsner 1988). But, the results of the debate were not conclusive. In order to estimate the correlation dimension correctly, we need a huge amount of data points but such a dataset is unfortunately not available (Grassberger 1986).

In this letter, we focus on the Arctic Oscillation Index (AOI) (Thompson and Wallace, 1998) and investigate its time series for the recent 60 years by employing surrogate data analysis. The Arctic Oscillation is the most dominant atmospheric variability in the Northern Hemisphere. Various characteristics of AOI are already known: for example, the variability of the AOI is coupled with the hydrosphere, cryosphere, and/or external driving forces (Feldstein 2002); AOI describes almost similar characteristics to the North Atlantic Oscillation (Feldstein and Franzke 2006); AOI is highly correlated with the intensity of the polar jet (Monahan and Fyfe 2008); when AOI was negative, the Arctic sea ice in summer decreased (Ogi and Yamazaki 2010); the transformed Eulerian mean circulation is related to the time scale for persistence of the AOI (Kidston et al. 2010); and the AOI in sea-level pressure is correlated with those of the surface air temperature in the Northern Hemisphere (Ohashi and Tanaka 2010).

We analyze the dataset of AOI with surrogate data analysis (Scheinkman and LeBaron 1989; Theiler et al. 1992; Schreiber and Schmitz 1996; Small et al. 2001). Surrogate data analysis is a kind of hypothesis testing used in nonlinear time series analysis. After developing a test statistic for surrogate data analysis, we show that AOI is nonlinear with determinism beyond pseudo-periodicity. Hence AOI is consistent with deterministic chaos and the dynamics can be predicted in a short-term but not in a long-term due to the sensitivity on initial conditions.

Corresponding author: Yoshito Hirata, Institute of Industrial Science, The University of Tokyo, 4-6-1 Komaba, Meguro-ku, Tokyo 153-8505, Japan. E-mail: yoshito@sat.t.u-tokyo.ac.jp. C2011, the Meteorological Society of Japan.

\section{Used dataset}

The Arctic Oscillation Index used in this study is the score time series of the first empirical orthogonal function of the barotropic component of the atmosphere (Tanaka 2003). The duration of the time series is from January 1950 to the mid of June 2010. The sampling interval is 6 hours. To this dataset, we applied surrogate data analysis.

\section{Surrogate data analysis}

In this section, we introduce surrogate data analysis, and develop and validate its test statistic.

The surrogate data analysis (Scheinkman and LeBaron 1989; Theiler et al. 1992; Schreiber and Schmitz 1996; Small et al. 2001) is a kind of hypothesis testing in nonlinear time series analysis. In the analysis, we first set a null-hypothesis. Second, we generate a number of surrogate datasets, i.e., random datasets that preserve the properties of the original dataset related to the nullhypothesis. Then, we compare the original dataset with the surrogate datasets with a test statistic. If the test statistic for the original dataset is sufficiently out of the range obtained from those of the surrogate datasets, then we reject the null-hypothesis. Otherwise, we cannot reject the null-hypothesis.

In the literature, mainly 4 types of surrogate datasets for a scalar time series have been proposed. The first type is random shuffle surrogates (Scheinkman and LeBaron 1989), where points of time series are exchanged randomly to destroy the dependence of points on their previous points, which we call serial dependence. Therefore, the first null-hypothesis is that there is no serial dependence. The second type is phase-randomized surrogates (Theiler et al. 1992), where the linear property, namely the power spectrum of the original data, is preserved but the phases are randomized. Hence, the second null-hypothesis is that the underlying dynamics is linear noise. The third type is iterative amplitude-adjusted Fourier transform surrogates (Schreiber and Schmitz 1996), where we preserve the distribution of points perfectly and the power spectrum the most. The third null-hypothesis is that the underlying dynamics is a nonlinear transformation of linear noise. Therefore, the second and third types of surrogates are for testing the nonlinearity of given datasets. The fourth type is pseudo-periodic surrogates, where local dynamics, and hence rough periodicity which we call pseudo-periodicity, is almost preserved but fine structures induced by determinism beyond pseudo-periodicity are destroyed. The fourth null-hypothesis is that there is no determinism beyond pseudo-periodicity (Small et al. 2001). If all these null-hypotheses are rejected, the underlying dynamics is consistent with deterministic chaos.

To compare an original dataset with its surrogate datasets, we need a test statistic. First, we used the Wayland statistic (Wayland et al. 1993), which has been often used in the literature of surrogate data analysis (see Hirata et al. (2007) and references therein for examples). The Wayland statistic tends to be close to 0 when the underlying dynamics is deterministic, and take a larger value close to 1 when it is stochastic. When we applied the Wayland statistic to the AOI data, although the first null-hypothesis was rejected (see Fig. 1), the second and third null-hypotheses were not rejected with the Wayland statistic. But, we also could obtain a 


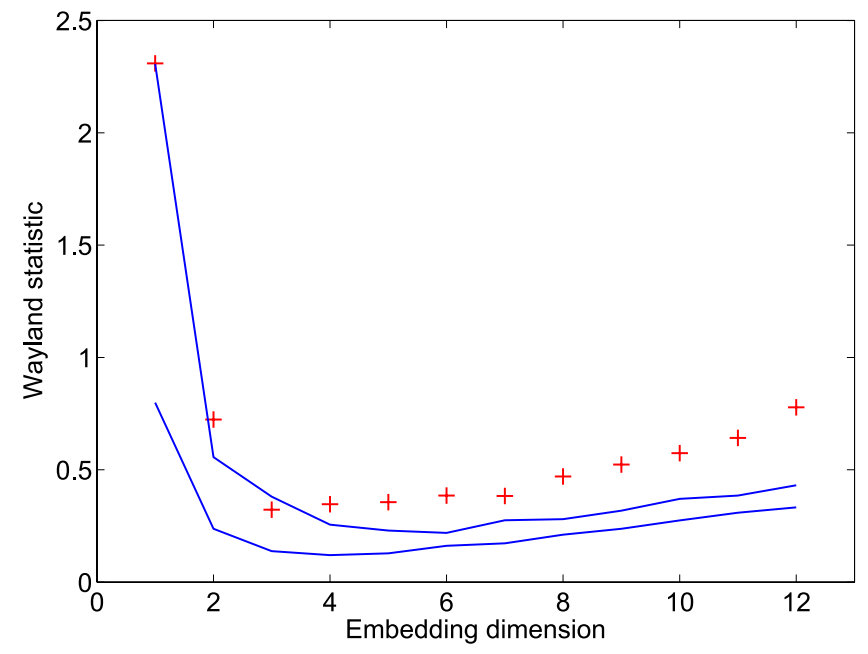

Fig. 1. Wayland statistic obtained from the original time series of AOI, which is denoted by symbol + . The maximum and minimum of the Wayland statistic for 39 random shuffle surrogates are also shown with solid lines.

hint of the underlying dynamics from these results since the direction of the rejection for the first null-hypothesis was opposite, i.e., the value for the original time series was greater than those for its random shuffle surrogates (Hirata et al. 2007). This is a typical characteristic for high-dimensional systems. There is a possibility that since the Wayland statistic was designed for identifying determinism in a low-dimensional space, the second and the third nullhypotheses were not rejected in this case of AOI. This reasoning motivated us to define a new test statistic.

Let us modify a recently defined statistic (Marwan et al. 2009) based on recurrence plots. Recurrence plots (Eckmann et al. 1987; Marwan et al. 2007) are originally two-dimensional graphs for visualizing time series data. Suppose that a scalar time series $\{s(i)$ $\in R: i=1,2, \ldots, n\}$ is given. Then, to reconstruct original states of the underlying dynamics from a scalar time series, we employ delay coordinates $z(i)=(s(i), s(i+1), \ldots, s(i+(m-1)))$, where $m$ is called as the embedding dimension. Let $d$ be the function defining the Euclidean distance in $R^{m}$. Then, a recurrence plot is defined as

$$
R(i, j)= \begin{cases}1, & \text { if } d(z(i), z(j))<r \\ 0, & \text { otherwise }\end{cases}
$$

When $R(i, j)=1$, we plot a dot at $(i, j)$. Otherwise, we do not plot anything at $(i, j)$. Since recurrence plots can contain almost all information of time series except for the spatial scale (Hirata et al. 2008a), recurrence plots are good tools for representing time series data.

One of the recent trends of research on recurrence plots is to regard a recurrence plot as a network and quantify the characteristics of the network (Marwan et al. 2009). Suppose that there are $n-m+1$ nodes labelled from 1 to $n-m+1$. If $R(i, j)=1$, then nodes $i$ and $j$ are connected by an edge. Nodes $i$ and $j$ are not connected by an edge if $R(i, j)=0$. When $i=j$, we ignore $R$ and do not consider the self-loops. Therefore, the adjacency matrix $A(i, j)$ for the network is defined as $A(i, j)=R(i, j)-\delta(i, j)$, where $\delta$ is the Kronecker delta, i.e.,

$$
\delta(i, j)=\left\{\begin{array}{l}
1, \text { if } i=j, \\
0, \text { otherwise. }
\end{array}\right.
$$

Marwan et al. (2009) used a set of statistics, one of which is the clustering coefficient. Let $k_{v}$ be the number of neighbors for node $v$, which is defined by

$$
k_{v}=\sum_{i=1}^{n} A(v, i)
$$

The local clustering coefficient for node $v$ is defined by

$$
C_{v}=\frac{\sum_{i, j=1}^{n} A(v, i) A(i, j) A(j, v)}{k_{v}\left(k_{v}-1\right)} .
$$

This quantity is the probability that two neighbors of $v$ are connected with an edge, hence $0 \leq C_{v} \leq 1$ as long as $k_{v}>1$. The clustering coefficient $C$ is the following average of the local clustering coefficients:

$$
C=\frac{1}{n} \sum_{v=1}^{n} C_{v}
$$

The clustering coefficient is considered to be related to the dimension of a system since the system dimension becomes higher, then $C_{v}$, namely the probability that two neighbors are connected with an edge, becomes lower.

When we try to apply the clustering coefficient to surrogate data analysis, we face a problem. For some time series, the number $k_{v}$ of neighbors for node $v$ becomes 0 or 1 , which makes the $C_{v}$ infinite, hence $C$ as well. To overcome this problem, we define the modified local clustering coefficient for $v$ by

$$
\tilde{C}_{v}=\frac{\sum_{i, j=1}^{n} A(v, i) A(i, j) A(j, v)+0.5}{k_{v}\left(k_{v}-1\right)+0.5} .
$$

This modification makes $\tilde{C}_{v}$ vary between 0 and 1 even if $k_{v}=$ 0 or 1 . This kind of modification is often used in information theory as the Krishevsky-Trofimov estimator (Willems et al. 1995). Using this $\tilde{C}_{v}$, we define the modified clustering coefficient $\tilde{C}$ by

$$
\tilde{C}=\frac{1}{n} \sum_{v=1}^{n} \tilde{C}_{v}
$$

As discussed above, $\tilde{C}$ is considered to be related to the system dimension: If the system dimension is larger, then $\tilde{C}_{v}$ becomes smaller, which makes $\tilde{C}$ smaller as well. The advantage of $\tilde{C}$ over the correlation dimension is that $\tilde{C}$ can be calculated straightforward and objectively; on the other hand, the estimation of the correlation dimension mostly contains a step of finding a scaling region, which can be subjective.

First, we tested that the new test statistic $\tilde{C}$ works correctly using toy models. Here we use five different toy models: white noise, an auto-regressive linear (AR) model, the Rössler model with a stable periodic orbit, the Lorenz' 63 model with a chaotic orbit, and the Lorenz'96 model.

The white noise is defined by

$$
y(i)=\eta_{i},
$$

where $\eta_{i}$ follows the Gaussian distribution of mean 0 and standard deviation 1 .

The AR model is defined by

$$
y(i+1)=-0.7 y(i)+\eta_{i} .
$$

As for the Rössler model (Rössler 1976) with a stable periodic orbit, we used the following equations to generate a time series:

$$
\begin{aligned}
& \dot{y}_{1}=-\left(y_{2}+y_{3}\right), \\
& \dot{y}_{2}=y_{1}+0.2 y_{2}, \\
& \dot{y}_{3}=0.4+y_{3}\left(y_{1}-4.5\right) .
\end{aligned}
$$

Here, we used $y_{1}$ observed every 0.1 unit time. 
Table 1. Results of surrogate data analysis. In this table, each column shows the results of different null-hypotheses, namely, the first, the second, the third, and the fourth null-hypotheses mentioned in the main text. Symbol $\times$ means that the null-hypothesis was not rejected and $\checkmark$ means that the null-hypothesis was rejected.

\begin{tabular}{lcccc}
\hline \multicolumn{1}{c}{ time series } & first & second & third & fourth \\
\hline white noise & $\times$ & $\times$ & $\times$ & $\times$ \\
AR model & $\checkmark$ & $\times$ & $\times$ & $\times$ \\
periodic Rössler & $\checkmark$ & $\checkmark$ & $\checkmark$ & $\times$ \\
chaotic Lorenz'63 & $\checkmark$ & $\checkmark$ & $\checkmark$ & $\times$ \\
Lorenz'96 & $\checkmark$ & $\checkmark$ & $\checkmark$ & $\checkmark$ \\
\hline \multicolumn{1}{c}{ AOI } & $\checkmark$ & $\checkmark$ & $\checkmark$ & $\checkmark$ \\
\hline
\end{tabular}

The Lorenz'63 model (Lorenz 1963) is a typical model of low-dimensional chaos. We used the following equations:

$$
\begin{aligned}
& \dot{y}_{1}=-10\left(y_{1}-y_{2}\right), \\
& \dot{y}_{2}=-y_{1} y_{3}+28 y_{1}-y_{2}, \\
& \dot{y}_{3}=y_{1} y_{2}-\frac{8}{3} y_{3} .
\end{aligned}
$$

In this model, we observed $y_{1}$ every 0.01 unit time.

The Lorenz'96 model (Lorenz 1996) is in a 240-dimensional state space and is a model of high-dimensional chaotic atmosphere. In the Lorenz'96 model, there are the number $I$ of large scale variables $x_{i}$ and the number $I \times J$ of small scale variables $y_{j, i}$. Using these variables, the equations are defined as

$$
\begin{aligned}
& \dot{x}_{i}=x_{i-1}\left(x_{i+1}-x_{i-2}\right)-x_{i}+F-\frac{h_{x} c}{b} \sum_{j=1}^{J} y_{j, i}, \\
& \dot{y}_{j, i}=c b y_{j+1, i}\left(y_{j-1, i}-y_{j+2, i}\right)-c y_{j, i}+\frac{h_{y} c}{b} x_{i},
\end{aligned}
$$

where we used the following cyclic boundary conditions:

$$
x_{I+i}=x_{i}, \quad y_{j+J, i}=y_{j, i+1}, \quad y_{j-J, i}=y_{j, i-1} .
$$

We used $I=40, J=5, F=8, b=10, c=10, h_{x}=1$, and $h_{y}=1$. We observed $y_{1,1}$ every 0.05 unit time to obtain a scalar time series.

From each of the models, we generated a scalar time series of length 5518 and applied surrogate data analysis with the test statistic $\tilde{C}$. Let $\{s(i)\}$ be a scalar time series generated from one of the models. First, we cut both ends of each time series so that the changes at the end points do not produce artificial high frequency components (Schreiber and Schmitz 2000). Second, we used delay coordinates to define $z(i)=(s(i), s(i+1), \ldots, s(i+(m-1)))$. We varied $m$ between 1 and 20. Then, we obtained recurrence plots using thresholds such that the recurrence rate became 0.05 . For each null-hypothesis, we generated 39 of its surrogates, which made the significance level $5 \%$ for each $m$. We declared that a null-hypothesis is rejected with the significance level of $2 \%$ if the $\tilde{C}$ for the original time series is out of the range of those for the surrogate datasets in more than 3 different $m$ 's.

The results are summarized in Table 1 (see also Fig. 2 for the results of the Lorenz'96 model). The datasets of the white noise, the AR model, the periodic Rössler model, and the Lorenz'96 model generated the results we expected. As for the time series generated from the chaotic Lorenz'63 model, the fourth nullhypothesis was not rejected. This implies that the new test statistic $\tilde{C}$ is sensitive only when the system is in a high-dimensional state space.

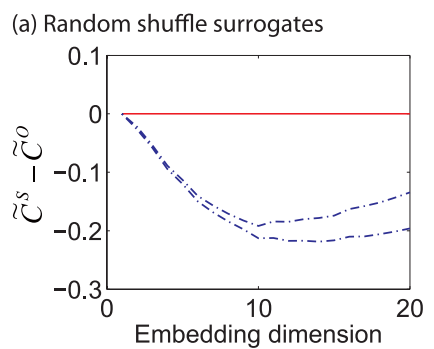

(b) Phase-randomized surrogates

(c) IAAFT surrogates
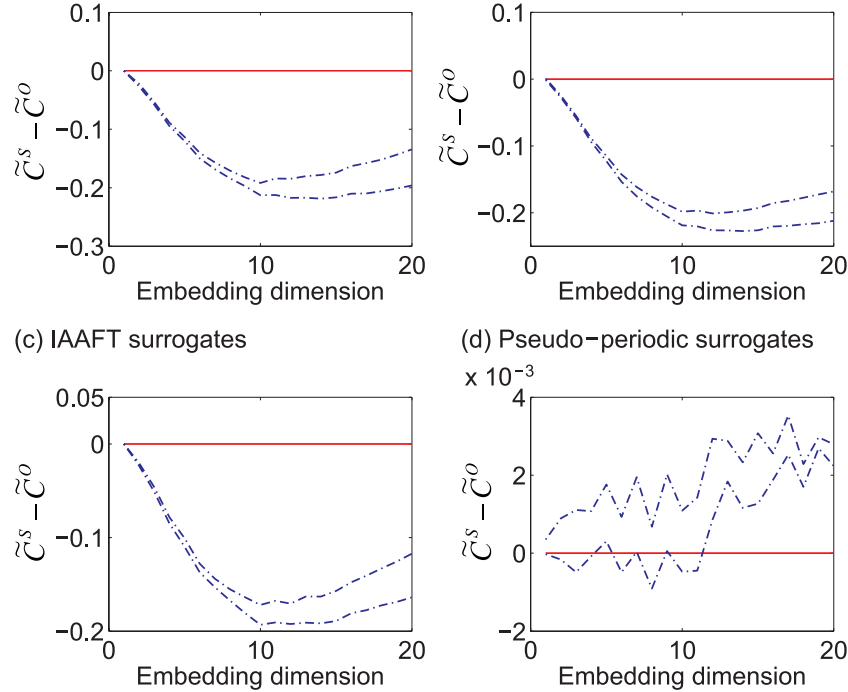

(d) Pseudo-periodic surrogates $\times 10^{-3}$

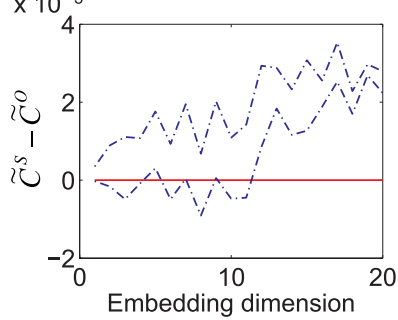

Fig. 2. Results of the surrogate data analysis for the dataset generated from the Lorenz'96 model with the newly proposed test statistic. (a) Random shuffle surrogates based on the first null-hypothesis. (b) Phase-randomized surrogates based on the second null-hypothesis. (c) Iterative amplitude adjusted Fourier transform (IAAFT) surrogates based on the third nullhypothesis. (d) Pseudo-periodic surrogates based on the fourth nullhypothesis. In each panel, the vertical axis shows the difference $\tilde{C}^{S}-\tilde{C}^{O}$ between the test statistic $\widetilde{C}^{S}$ obtained from a surrogate dataset and the test statistic $\tilde{C}^{O}$ obtained from the original data, i.e., the dash-dotted lines are the minimum and the maximum of $\tilde{C}^{S}-\tilde{C}^{O}$.
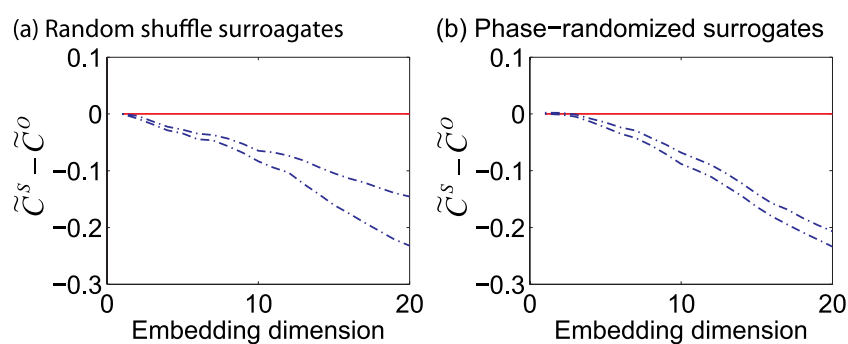

(c) IAAFT surrogates

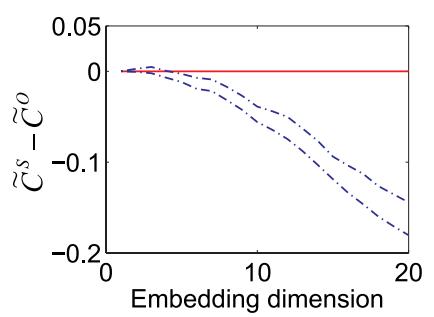

(d) Pseudo-periodic surrogates

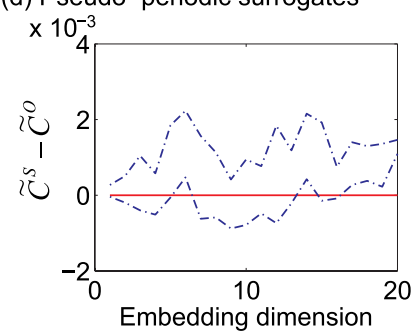

Fig. 3. Results of the surrogate data analysis for the dataset of AOI. See the caption of Fig. 2 to find how to read this figure.

\section{Application to the Arctic Oscillation Index}

Finally, we applied the proposed test statistic $\tilde{C}$ to the time series of AOI. As preprocessing, we sub-sampled the dataset every 4 days, which made the length of time series 5518. Then, we confirmed the stationarity of the dataset using the method of Kennel (1997). Except for this, the rest was the same as the cases of the above toy models. The results, shown in Table 1 and Fig. 3, were similar to those of Lorenz'96 model, implying that they are consistent with high-dimensional deterministic chaos. 


\section{Summary and discussions}

To further characterize the dataset of the Arctic Oscillation Index (AOI) as a nonlinear time series, we tried to estimate the maximal Lyapunov exponent. Although we observed that the distances between nearby orbits tended to diverge almost exponentially, we failed to obtain a consistent value when sub-sampling the dataset. It is possibly due to the dimension of AOI. We also confirmed that AOI of the next day can be predicted from the previous AOIs better by nonlinear time series prediction than the persistent prediction (Hirata et al. 2008b), under which, in this case, the prediction of the AOI for the next day is provided by the current value of the AOI.

Our results are significantly different from the debate held mostly in 1980s since our test statistic, which can be obtained objectively, is specially tuned for high-dimensional systems.

The overall results imply that the underlying dynamics of AOI is of deterministic chaos and is not white noise. Thus, the dynamics may be predictable in a short-term but not in a long-term due to the sensitive dependence of initial conditions.

\section{Acknowledgments}

The present work was partly supported by the Japan Society for the Promotion of Science (JSPS) through the Funding Program for World-Leading Innovative R\&D on Science and Technology (FIRST Program).

\section{References}

Eckmann, J. P., S. O. Kamphorst, and D. Ruelle, 1987: Recurrence plots of dynamical systems. Europhys. Lett., 4, 973-977.

Essex, C., T. Lookman, and M. A. H. Nerenberg, 1987: The climate attractor over short timescales. Nature, 326, 64-66.

Feldstein, S. B., 2002: The recent trend and variance increase of the annular mode. J. Climate, 15, 88-94.

Feldstein, S. B., and C. Franzke, 2006: Are the north Atlantic oscillation and the northern annular mode distinguishable? $J$. Atmos. Sci., 63, 2915-2930.

Grassberger, P., 1986: Do climatic attractors exist? Nature, 323, 609-612.

Grassberger, P., 1987: Grassberger replies. Nature, 326, 524.

Hirata, Y., S. Horai, H. Suzuki, and K. Aihara, 2007: Testing serial dependence by Random-shuffle surrogates and the Wayland method. Phys. Lett. A, 370, 265-274.

Hirata, Y., S. Horai, and K. Aihara, 2008a: Reproduction of distance matrices and original time series from recurrence plots and their applications. Eur. Phys. J. Special Topics, 164, 1322.

Hirata, Y., D. P. Mandic, H. Suzuki, and K. Aihara, 2008b: Wind direction modelling using multiple observation points. Phil. Trans. R. Soc. A, 366, 591-607.

Kennel, M. B., 1997: Statistical test for dynamical nonstationarity in observed time-series data. Phys. Rev. E, 56, 316-321.

Kidston, J., D. M. W. Drierson, J. A. Renwick, and G. K. Vallis, 2010: Observations, simulations, and dynamics of jet stream variability and annular modes. J. Climate, 23, 6186-6199.

Lorenz, E. N., 1991: Dimension of weather and climate attractors. Nature, 353, 241-244.

Lorenz, E. N., 1996: Predictability: A problem partly solved. Proc. Seminar on Predictability, 1, 1, ECMWF, Reading, Berkshire, UK.

Marwan, N., M. C. Romano, M. Thiel, and J. Kurths, 2007: Recurrence plots for the analysis of complex systems. Phys. Rep., 438, 237-329.

Marwan, N., J. F. Donges, Y. Zou, R. V. Donner, and J. Kurths, 2009: Complex network approach for recurrence analysis of time series. Phys. Lett. A, 373, 4246-4254.

Monahan, A. H., and J. C. Fyfe, 2008: On annular modes and zonal jets. J. Climate, 21, 1963-1978.

Nicolis, C., and G. Nicolis, 1984: Is there a climatic attractor? Nature, 311, 529-532.

Nicolis, C., and G. Nicolis, 1987: Evidence for climatic attractors. Nature, 326, 523.

Ogi, M., and K. Yamazaki, 2010: Trends in the summer northern annular mode and Arctic sea ice. Sci. Online Lett. Atmos., 6, 41-44.

Ohashi, M., and H. L. Tanaka, 2010: Data analysis of recent warming pattern in the Arctic. Sci. Online Lett. Atmos., 6A, $1-4$.

Rössler, O. E., 1976: An equation for continuous chaos. Phys. Lett. A, 57A, 397-398.

Scheinkman, J. A., and B. LeBaron, 1989: Nonlinear dynamics and stock returns. J. Bus., 62, 311-337.

Schreiber, T., and A. Schmitz, 1996: Improved surrogate data for nonlinear tests. Phys. Rev. Lett., 80, 2105-2108.

Schreiber, T., and A. Schmitz, 2000: Surrogate time series. Physica D, 142, 346-382.

Small, M., D. Yu, and R. G. Harrison, 2001: Surrogate test for pseudoperiodic time series. Phys. Rev. Lett., 87, 188101.

Tanaka, H. L., 2003: Analysis and modeling the Arctic Oscillation using a simple barotropic model with baroclinic eddy forcing. J. Atmos. Sci., 60, 1359-1379.

Theiler, J., S. Eubank, A. Longtin, B. Galdrikian, and J. D. Farmer, 1992: Testing for nonlinearity in time-series: the method of surrogate data. Physica D, 58, 77-94.

Thompson, D. W. J., and J. M. Wallace, 1998: The Arctic Oscillation signature in the wintertime geopotential height and temperature fields. Geophys. Res. Lett., 25, 1297-1300.

Tsonis, A. A., and J. B. Elsner, 1988: The weather attractor over very short timescales. Nature, 333, 545-547.

Wayland, R., D. Bromley, D. Pickett, and A. Passamante, 1993: Recognizing determinism in a time series. Phys. Rev. Lett., 70, 580-582.

Willems, F. M. J., Y. M. Shtarkov, and T. J. Tjalkens, 1995: The context-tree weighting method: Basic properties. IEEE Trans. Inf. Theory, 41, 653-664.

Manuscript received 8 December 2010, accepted 20 February 2011 SOLA: http://www.jstage.jst.go.jp/browse/sola 